\title{
ULF wave measurements onboard the Interball auroral probe
}

\author{
S. Perraut ${ }^{1}$, A. Roux ${ }^{1}$, F. Darrouzet ${ }^{1}$, C. de Villedary ${ }^{1}$, M. Mogilevsky ${ }^{2}$, F. Lefeuvre ${ }^{3}$ \\ ${ }^{1}$ CETP/UVSQ, 10-12 Av. de l'Europe, F-78140 Velizy, France \\ Fax: +1/39 2549 22; e-mail: sylvaine.perraut@cetp.ipsl.fr \\ ${ }^{2}$ Space Research Center, Profsoyuznaya 84/32, Moscow, Russia \\ ${ }^{3}$ LPCE, 3A, Av. de la Recherche Scientifique, F-45071 Orleans cedex 2, France
}

Received: 22 September 1997 / Revised: 27 February 1998 / Accepted: 10 March 1998

\begin{abstract}
The IESP experiment implemented onboard the Interball auroral probe measures the six components $(3 B, 3 E)$ of the waves in the ULF range: $0.1-10 \mathrm{~Hz}$ and from time to time $0-30 \mathrm{~Hz}$. Two different kinds of waves have been observed in the auroral region at altitudes between 10000 and $20000 \mathrm{~km}$ : (1) electrostatic emissions which consist of quasi-monochromatic structures with frequencies above the oxygen gyrofrequency, superimposed on a wide band signal interpreted as a Doppler broadening, (2) electromagnetic wide band spectrum fluctuations. These emissions are interpreted as current-driven electromagnetic or electrostatic ion cyclotron waves. The electromagnetic/electrostatic character is controlled by the plasma parameter $\beta_{i}$ and by the $\mathrm{O}^{+}$concentration.
\end{abstract}

Key words. Magnetospheric physics · Auroral phenomena $\cdot$ Plasma waves and instabilities · Interball Auroral probe

\section{Introduction}

The auroral region above the ionosphere at an altitude of $\geq 2$ Earth radii has already been investigated by the American satellites S3-3 and DE-1 and by the Swedish satellite Viking. One of the most interesting results of these missions was to show that acceleration processes take place in this altitude range. The analysis of the observed electron and ion distributions has given evidence of an upward-directed parallel electric field, accelerating electrons downward and ions upward (see for instance Louarn et al., 1990). Quasi-static parallel electric fields were also measured and interpreted in terms of large-scale accelerating structures (Lindqvist

Correspondence to: Dr. S. Perraut and Marklund, 1990), and/or small-scale electrostatic structures, solitary waves and double layers (Mozer and Temerin, 1983; Boström et al., 1988). While most of the waves and structures observed were interpreted as being electrostatic, in some cases the authors have concluded the existence of electromagnetic waves, generated by wave-particle interactions (Temerin and Lysak, 1984; Gustafsson et al., 1990). The investigation of S3-3 and Viking in this low-frequency domain, however, has been restricted by the absence of, or lack of sensitivity range of the magnetic sensors carried onboard in the $0-10 \mathrm{~Hz}$ frequency. For instance on Viking the magnetic loop was designed to make measurements above a few tens of $\mathrm{kHz}$.

It is well known however, from theoretical studies conducted by Hasegawa and Uberoi (1982) and Goertz (1986), that electromagnetic waves with a finite parallel electric field, the so-called kinetic Alfvén waves (KAWs), can develop (Volokitin and Dubinin, 1989) over the auroral region and contribute to the parallel acceleration via their parallel electric field. The IESP experiment which carries a complete set of instruments for measuring the components of the electromagnetic waves in the ULF range up to $30 \mathrm{~Hz}$ is particularly well suited to disentangle the electromagnetic and electrostatic waves and therefore to assess the potential role of KAWs at accelerating particles. The trajectory of the Interball Auroral probe is well adapted to exploring the region along the auroral field lines where the different mechanisms of particle acceleration occur since it covers altitude ranges between 10000 and $20000 \mathrm{~km}$.

In the first part we will describe the experiment. Typical observations will be presented in the second section. A tentative interpretation of the phenomena observed in the regions probed by Interball will be given in the third part.

\section{Description of the experiment}

The IESP experiment (devoted to the measurement of the waves in the ULF range) is part of a wider wave 
complex covering a frequency range from DC to $2 \mathrm{MHz}$. Basically, the IESP experiment has been designed for measuring: (1) the waveform of the six components of the electromagnetic field in the frequency ranges from 0.1 to 10 or $30 \mathrm{~Hz},(2)$ the three components of the DC electric field (1 sample/s) and (3) the electric potential of the satellite (1 sample/16s) which is the voltage measured between one of the spheres and the structure of the satellite. This last information is transmitted to the RON (spacecraft potential control) experiment which controls an ion gun to neutralize the ambient plasma (Riedler et al., 1995).

The magnetic components are measured with the triaxial search coil magnetometer designed for the lower ULF/ELF range of the MEMO (Mesure Multicomposante des Ondes, in English Multicomponent Wave Measurement) experiment; it detects waves from $0.1 \mathrm{~Hz}$ to $1 \mathrm{kHz}$ (see Lefeuvre et al., 1995). The magnetic signals come from MEMO to IESP through the $\mathrm{NVKONCH}$ experiment (devoted to the measurement of the waves in the ELF/VLF frequency range) which also covers the frequency range from $5 \mathrm{~Hz}$ to $22 \mathrm{kHz}$. The electric field is measured with six spheres $(80 \mathrm{~mm}$ diameter) mounted at the ends of long booms. The longest booms ( $25 \mathrm{~m}$ tip to tip) are in the spin plane; the pair ED1 and ED2 corresponds to $E_{y}$ and ED3 and ED4 to $E_{z}$; the third pair of booms (ED5-ED6) is about $4.5 \mathrm{~m}$ long corresponding to $E_{x}$ more or less aligned along the spin axis (ED5 makes an angle of $6^{\circ}$ with respect to the spin axis in order to avoid shadowing effects on ED6). The IESP instrument provides electric signals to the other wave experiments $\mathrm{NVKONCH}$ and MEMO.

The main characteristics of the instrument are as follows:

search coil sensitivity: $0.2 \mathrm{pT} . \mathrm{Hz}^{-1 / 2}$ at $10 \mathrm{~Hz}$; electric sensitivity: $0.5 \mathrm{mV} / \mathrm{m}$ for the DC, $3.10^{-5} \mathrm{~V} \cdot \mathrm{m}^{-1} \cdot \mathrm{Hz}^{-1 / 2}$ at $1 \mathrm{~Hz}$ and $10^{-6} \mathrm{~V} \cdot \mathrm{m}^{-1} \cdot \mathrm{Hz}^{-1 / 2}$ at $10 \mathrm{~Hz}$; dynamic range: the waveforms of the $\mathrm{AC}$ components are digitized with 12 bits, i.e., $72 \mathrm{~dB}$, with four different gain levels from -6 to $+30 \mathrm{~dB}$, the highest gain corresponding to $0.062 \mathrm{mV} /$ bin.

The transmission of the AC components needing a large amount of telemetry for the six components, different modes have been defined depending upon the telemetry allocation. IESP has the choice between the two telemetry systems: SSNI (Scientific Information Collection System) and STO. For SSNI this telemetry is organized in packets of 960 bits. With 8 packets/s, IESP transmits 6 channels up to $30 \mathrm{~Hz}$ (sampling frequency: $69.189 \mathrm{~Hz}$ ); with 4 packets/s, IESP transmits 6 channels up to $10 \mathrm{~Hz}$ (sampling frequency: $24.615 \mathrm{~Hz}$ ); and with 2 packets/s IESP can choose 4 channels out of the 6 channels and covers up to $10 \mathrm{~Hz}$.

As regards STO, depending upon the quality of the transmission from the spacecraft to the ground, the telemetry rate can vary between $40,20,10$, and $5 \mathrm{kbit} / \mathrm{s}$. Furthermore IESP can get three different telemetry allocations: 84,48 , or 42 bytes/format (of 512 bytes). The IESP data transmission capability with STO are summarized in the Table 1.
Table 1. IESP data transmission capability with STO

\begin{tabular}{|c|c|c|c|}
\hline $\begin{array}{l}\text { TM rate } \\
\mathrm{kb} / \mathrm{s}\end{array}$ & $\begin{array}{l}\text { frame } \\
\text { duration }\end{array}$ & \multicolumn{2}{|c|}{$\begin{array}{l}\text { data transmission capability } \\
\text { components-bandwidth }\end{array}$} \\
\hline 40 & 0.1 & 6 comp $-30 \mathrm{~Hz}$ & 6 comp-10 Hz \\
\hline 20 & 0.2 & 6 comp $-10 \mathrm{~Hz}$ & 4 comp-10 Hz \\
\hline 10 & 0.3 & 4 comp $-10 \mathrm{~Hz}$ & 2 comp-10 Hz \\
\hline 5 & 0.4 & $\begin{array}{l}2 \text { comp }-10 \mathrm{~Hz} \\
84 \\
\text { telemetry alloca }\end{array}$ & $\begin{array}{l}1 \text { comp-10 Hz } \\
48 \text { or } 42 \\
\text { byte/frame }\end{array}$ \\
\hline
\end{tabular}

The data can be transmitted either directly to the ground or after recording on board. The control of the operation modes (filters at 10 or $30 \mathrm{~Hz}$ and channel selection) and of the gain is made by digital telemetry commands (UKS).

The IESP experiment has no specific internal calibration system. However, IESP is interconnected with MEMO through NVKONCH and receives a calibration signal from MEMO for the magnetic components.

\section{Observations}

The Interball Auroral probe was launched successfully on 29 August 1996. The spin period of this satellite is 2 min. During a period of about 3 months the IESP experiment has recorded regularly the waveforms of the six components of the electromagnetic waves up to 10 or $30 \mathrm{~Hz}$ when Interball was flying across the auroral region or the polar cap at an altitude between 10000 and $20000 \mathrm{~km}$. After 20 December 1996, only the electric signals have been transmitted to the ground, since the IESP experiment did not receive the magnetic components from the MEMO via NVKONCH experiments. A survey of the events detected during this timeinterval allowed us to distinguish different classes of emissions in the ULF frequency domain. Notice that the proton gyrofrequency was in average around $15 \mathrm{~Hz}$, which is above the range covered by the IESP experiment during most of the time-periods. Essentially, two categories of waves have been identified from this survey: (1) electrostatic waves (2) electromagnetic fluctuations with a wide frequency spectra extending up to $30 \mathrm{~Hz}$. These waves are observed in the auroral region, when strong fluxes of electrons and ions in the energy range between 1 and $10 \mathrm{keV}$ are detected.

\subsection{Electrostatic waves}

The spectrograms of the electric components displayed in Plate 1 show a typical example of electrostatic waves. The parallel component corresponds to the signal detected with the spheres almost aligned with the spin axis, $X$; the perpendicular component is a combination of the two components $Y$ and $Z$ in the spin plane. The same referential applies for the magnetic components which are not plotted for this event. These waves have 


\begin{tabular}{|lllll}
\hline INTERBALL-AURORAL & 963012 2_S2_1 & IESP & (CETP/CNES)
\end{tabular}

$$
\text { Day : 22/Oct/1996 } \begin{array}{r}
\text { Orbit }: 228 \\
\text { E/B }: 0-10 \mathrm{~Hz}
\end{array}
$$

Telemesure SSNI

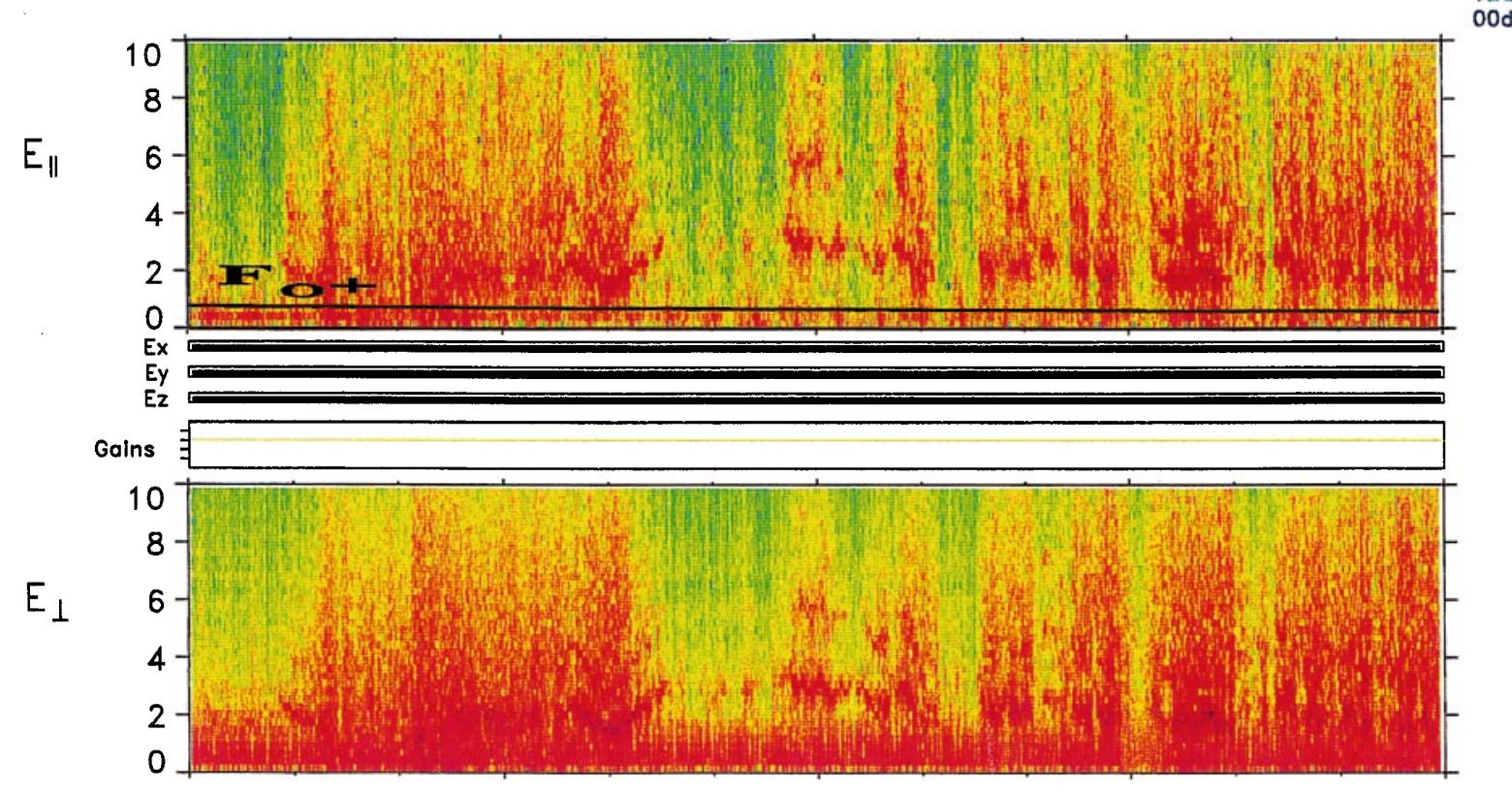

UT 21:00 21:05 21:10 21:15 21:20 21:25 21:30 21:35 21:40 21:45 21:50 21:55 21:59

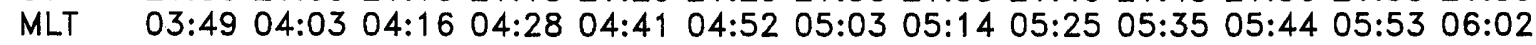

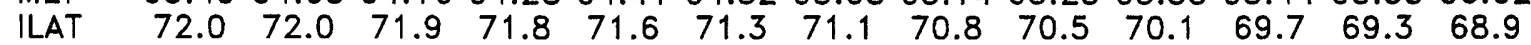

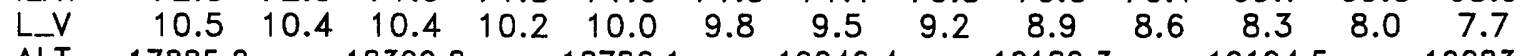

$\begin{array}{llllllll}\text { ALT } & 17885.2 & 18399.8 & 18786.1 & 19046.4 & 19182.3 & 19194.5 & 19083.3\end{array}$

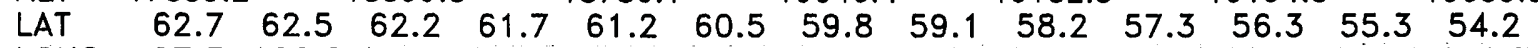

LONG $\quad 97.3 \quad 100.2103 .0105 .6108 .0110 .2112 .2114 .1115 .8117 .4118 .8120 .1121 .3$

Processing Data Version : v1 - Plotting Data Version : v1 - Date : 16/April/1997

Plate 1. Frequency-time spectrograms of the electric components. $E_{/ /}$ is parallel to the spin axis, $E$ is a composition of the two components $E_{Y}$ and $E_{Z}$ in the spin plane. The intensity of the waves is given by the color code on the right side. The black line superimposed on the upper spectrogram is the $\mathrm{O}^{+}$gyrofrequency $\mathrm{F}_{\mathrm{O}}+$. On $E_{\perp}$ the strong and

clear characteristics: the emission exhibits a discrete structure with a well-defined peak in amplitude but a frequency peak that varies with time; each structure consists of increasing or decreasing elements or a combination of these two kinds of elements forming a "chevron" pattern (see for example waves around 21:20 UT). The duration of these structured elements is typically of the order of $2 \mathrm{~min}$; sometimes a less intense harmonic structure is observed (for example around 21:43 UT). These waves are commonly observed in the auroral region at an altitude above $17000 \mathrm{~km}$ and in the morning sector between 03 and 06 local time. The long duration of such an event depends principally on the trajectory of Interball. During the event displayed in Plate 1, Interball stayed in the auroral region at local time favorable for these observations. These waves do regular signal below $1 \mathrm{~Hz}$ is an artefact which occur on $E_{Y}$ (see the waveform in Fig. 1). Notice the intense structured emissions above $\mathrm{F}_{\mathrm{O}}+$. At the same time a weaker signal spreads out on the whole frequency range

not present a significant magnetic component, as can be seen on the waveforms of the six components displayed in Figs. 1 and 2. The electric components are given in physical units, while the magnetic signals are given in telemetry units, because the transfer function of the magnetic antennas has not been applied at this stage of the study. The magnetic components $Y$ and $Z$ in the spin plane are modulated by the DC magnetic field component in this plane; this DC component varies between \pm 900 and $\pm 780 \mathrm{nT}$ from 21:00 to 22:00 UT. In the meantime, the $X$ component of the DC magnetic field decreases from 500 to $-40 \mathrm{nT}$. Then the DC magnetic field has a dominant component in the spin plane. The weak signal superimposed on the spin modulation corresponds to the noise of the instrument. The absence of significant signal on the magnetic 


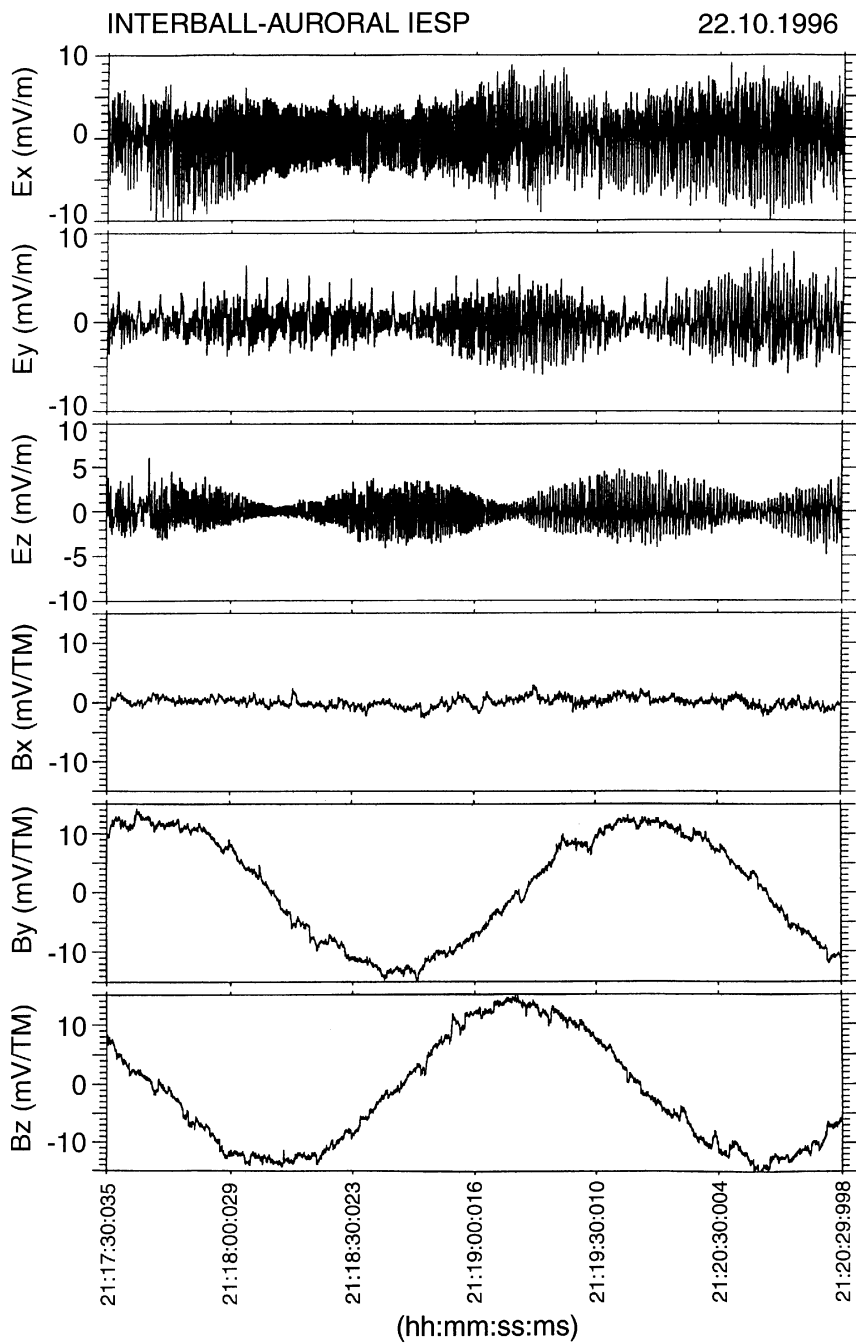

Fig. 1. Waveforms of the six components extract from the Plate 1 event; on the top the three electric and below the three magnetic. $E_{X}$ and $B_{X}$ are parallel to the spin axis. The spin period $T_{S}$ is $2 \mathrm{~min}$. The DC magnetic field $B_{\mathrm{O}}$ is large enough to induce a variation on the magnetic components of the search coil in the spin plane. The electric components exhibit a very monochromatic wave, not seen on the magnetic ones. The amplitude modulation of $E_{Y}$ and $E_{Z}$ at $T_{S} / 2$ points out that this wave electric field is perpendicular to $B_{\mathrm{O}}$

components leads us to conclude that these waves are electrostatic. Their amplitude reaches $20 \mathrm{mV} / \mathrm{m}$ peak to peak, around 21:48 UT (Fig. 2). Their frequency ranges between $2 \mathrm{~F}_{\mathrm{O}}+$ and $4 \mathrm{~F}_{\mathrm{O}}+$, i.e., 1.2 and $4 \mathrm{~Hz}$ on average since the magnetic field decreases from 1030 to $780 \mathrm{nT}$ from 21:00 to 22:00 UT $\left(\mathrm{F}_{\mathrm{O}}^{+}\right.$is the oxygen gyrofrequency). The waveforms exhibit clearly the effect of the rotation on the $E_{Y}$ and $E_{Z}$ components: each component has its maximum amplitude when the corresponding DC magnetic component is close to 0 . This is a strong indication that the electric field of the waves is perpendicular to the magnetic field. The waveforms displayed in Fig. 2 present intermittent fadings simultaneously on the three components of the electric field, around 21:47:30, 21:48:20, and 21:49:30.

Plate 1 gives evidence for a wide band signal covering most of the time the full frequency range, which is

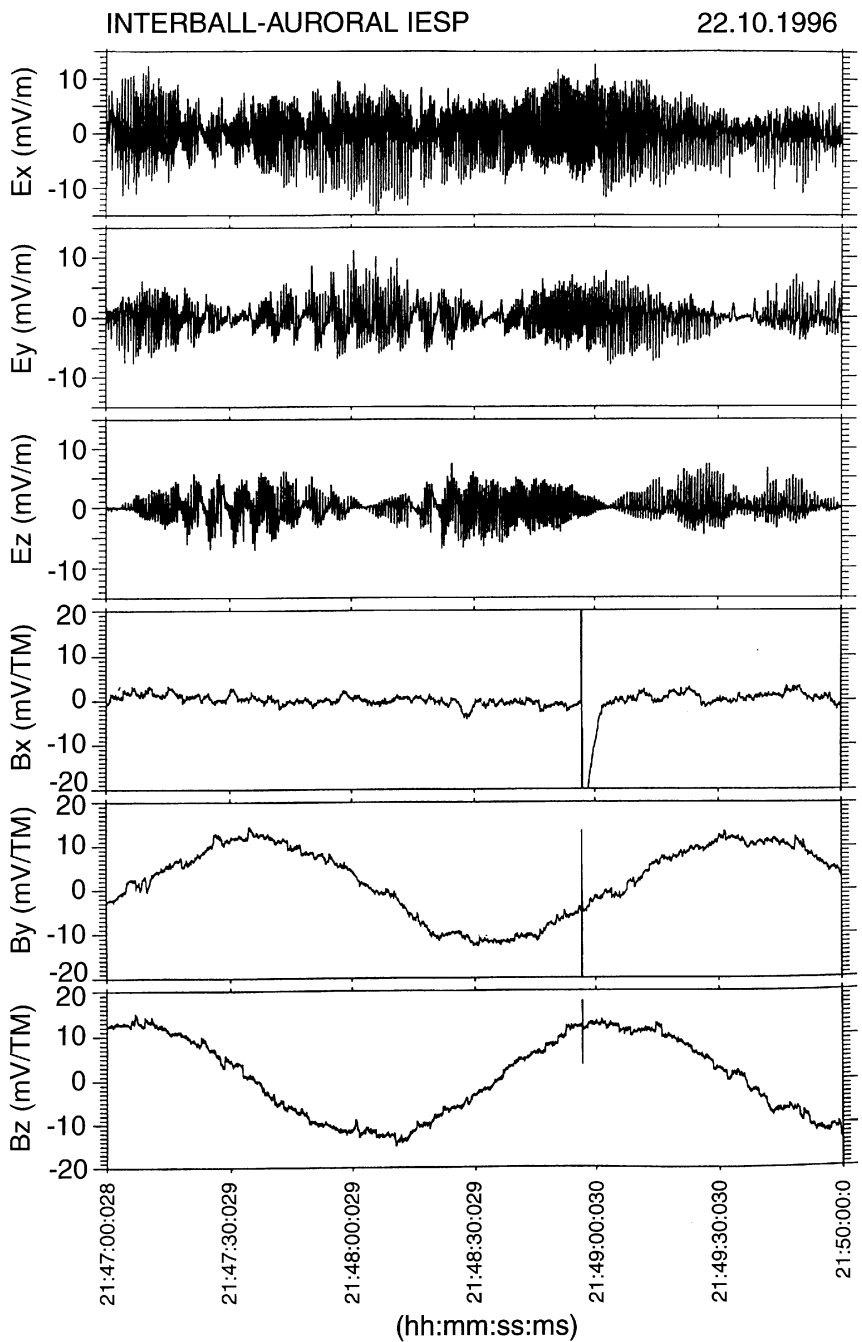

Fig. 2. Same as Fig. 1

superimposed on the quasi-monochromatic emissions already described. This wide band signal is generally less intense than the discrete structures superimposed. Consecutive spectra of the component $E_{X}$ are displayed (logarithmic scale) in Fig. 3. As soon as the discrete emission appears the spectrum widens. At 21:21:14, the emission detected around $2 \mathrm{~Hz}$ reaches $4(\mathrm{mV} / \mathrm{m})^{2} / \mathrm{Hz}$ and exhibits an harmonic structure and 2 min later the intensity of the signal decreases over the whole frequency range $\left(<0.1(\mathrm{mV} / \mathrm{m})^{2} / \mathrm{Hz}\right)$. This broadening of the spectrum in the presence of quasi-monochromatic structures could be explained by a Doppler-shift effect; this will be studied in Sect. 4. This kind of wide band fluctuation associated with discrete emissions differ from the ones described next.

\subsection{Electromagnetic fluctuations}

Waves with wide frequency spectra with no detectable peak in the frequency spectrum are also often detected when Interball crosses the auroral region. Plate 2 visualizes the spectrograms of the electric and magnetic 

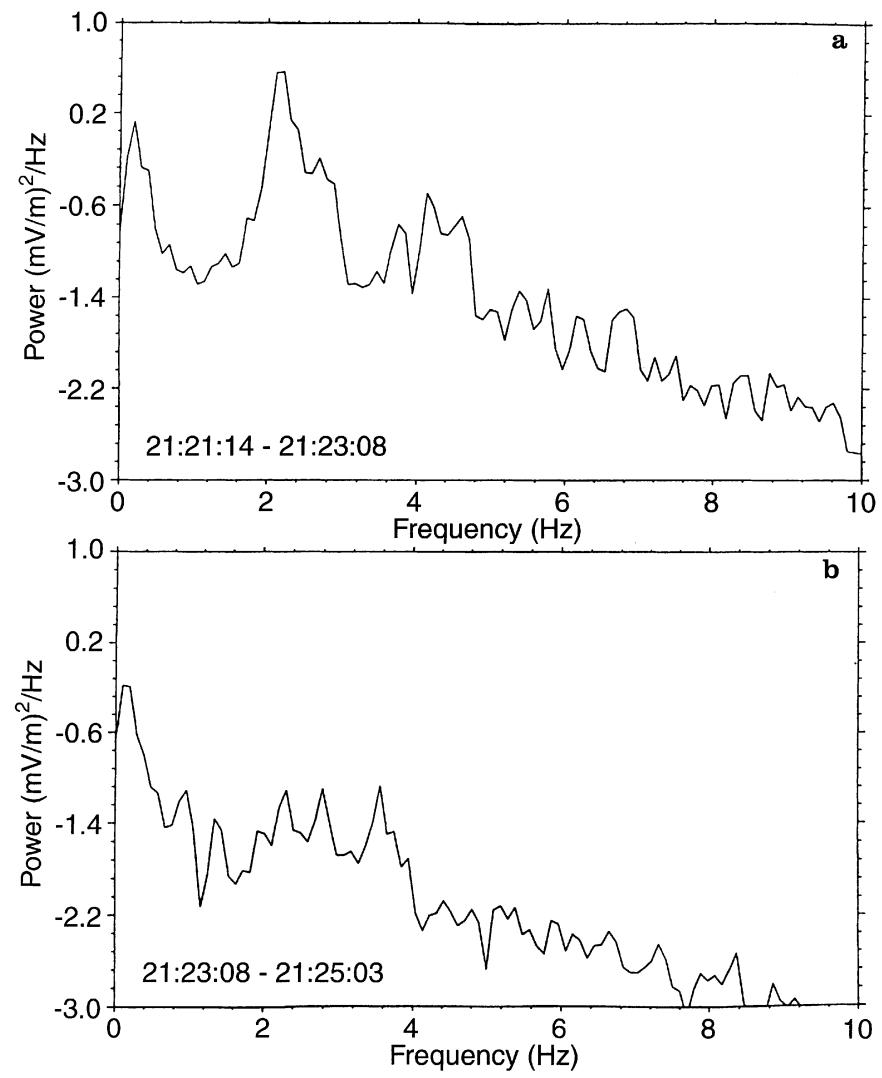

Fig. 3. Frequency spectra of the $E_{X}$ component at two different timeintervals. When the emission at $\sim 3 \mathrm{x} F_{O}^{+}$and its harmonic are observed the signal spreads out at high frequency (part a). $\Delta \mathrm{f} \sim 0.1 \mathrm{~Hz}$

components of a typical event detected when Interball flew over the polar cap and suddenly crossed a discrete auroral structure identified from the particle measurements (not shown here), between 15:12 and 15:22 UT. Notice that the sharp intensifications regularly spaced by $8 \mathrm{~min}$ and referenced with a letter "c" on the lower part of the spectrogram $E_{\perp}$ are artefacts due to the calibration of the NVKONCH experiment. The intensity of the fluctuations detected between 15:12 and 15:22 UT varies rapidly as a function of time. This kind of fluctuation requires a very careful analysis, because we know that the spectrum of a short lasting fluctuation can result in a wide band spectrum (see Louarn et al., 1994). Then it is necessary to examine in detail the waveform of these waves. The waveforms plotted in Fig. 4 give evidence of the bursty nature of the signal. The bursts occur in pair of wave packets spaced $40 \mathrm{~s}$ with a time repetition of the order of $2 \mathrm{~min}$. The increases in the amplitude of the electric and magnetic fluctuations are simultaneous at this time-scale (the strong signal observed before 15:17 UT seen especially on $E_{X}$ is due to the calibration of NVKONCH already mentioned).

The waveforms plotted with an enlarged time-scale in Fig. 5 show that for this event the fluctuations are observed at the same time on the six components. One also sees that the wave intensification occurs almost simultaneously over a broad range of frequencies for all components. Thus the enhancements seen on spectro- grams are not necessarily due to the short-lived structures observed from time to time, although they could contribute to it. When the electric and magnetic signals exhibit the same signature, as for example at 15:13:12 UT, it is possible to get a rough estimate of the ratio $\delta E / \delta \mathrm{B}$. At 15:13:12 $\mathrm{UT}$, it reaches $\sim 17 \times 10^{6} \mathrm{~m} / \mathrm{s}$; as the magnetic field is $\sim 1000 \mathrm{nT}$, the corresponding Alfvén velocity $V_{A}$ can be obtained by assuming that the density is about $1 \mathrm{~cm}^{-3}$; it is a typical value of the density over the auroral region explored by Interball. Then the value of the ratio $\delta E / \delta B$ measured from the waveforms compared to the local Alfvén velocity allows us to conclude that these waves are electromagnetic (Hasegawa and Uberoi, 1982).

The spike labelled 1 in Fig. 4 is also seen in Fig. 5. It lasts $\sim 2 \mathrm{~s}$ and is detected principally on $E_{X}, E_{Y}$ and $B_{Z}$. Its amplitude is $60 \mathrm{mV} / \mathrm{m}$ and $2.5 \mathrm{nT}$ for the electric and magnetic components, respectively. Then the ratio $\delta E /$ $\delta B$ is $24 \times 10^{6} \mathrm{~m} / \mathrm{s}$. It could be a solitary kinetic Alfvén wave (SKAW), as previously reported by Chmyrev et al. (1988) from Intercosmos-Bulgaria-1300 and Louarn et al. (1994) from Freja; these two studies, however, were made at a lower altitude. The spike labelled 2 has a larger amplitude; the $E_{X}$ component (which is not saturated since it comes from the shortest boom) reaches $200 \mathrm{mV} / \mathrm{m}$ p.p. and displays a signature on the $E_{X}$ component which is at least 3 times larger than spike 1; it is not clearly seen on the magnetic component, so its electromagnetic character is not granted. During this period the component of the DC magnetic field aligned along the spin axis is almost twice the perpendicular component; then the $X$-axis is more or less along $B_{0}$ and the signature of spike 2 could be the signature of a parallel electric field. A more systematic study of these spikes will be done in a future work.

A second event of this category was displayed on Plate 3. It has been detected when Interball was located at high invariant latitude $\left(\lambda \sim 74^{\circ}\right)$ and around midnight. The periods when the waves intensify correspond again to the presence of energetic particles which are the signature of the plasma sheet and map in the auroral region. Here again the fluctuations cover the whole frequency range and present very fast variations of the amplitude as a function of time. The waveforms of the measured components (Fig. 6) give evidence for weak amplitude electric components: less than $10 \mathrm{mV} / \mathrm{m}$ for the 5-s-period oscillations and much less for the higher frequencies. At $\sim 2 \mathrm{~Hz}$ the magnetic components reach $\sim 1 \mathrm{nT}$. The electromagnetic nature of these wide-band fluctuations varies with time. Figure 6 shows that between 21:33:00 and 21:36:30 the emission is electrostatic and after 21:36:30 it becomes electromagnetic. In fact most of the fluctuations displayed on Plate 3 are essentially electromagnetic, only the short-time interval

Plate 2. Same as Plate 1. Same definition for $B_{/ /}$and $B_{\perp}$. Regular intensifications of the signal referenced as $\mathrm{c}$ result of the calibration of another wave instrument NVKONCH. Wide band electromagnetic fluctuations are clearly seen between 21:12 and 21:22 UT. Notice the bursty or filamentary nature of these emissions. The signal at low frequency has the strongest amplitude 

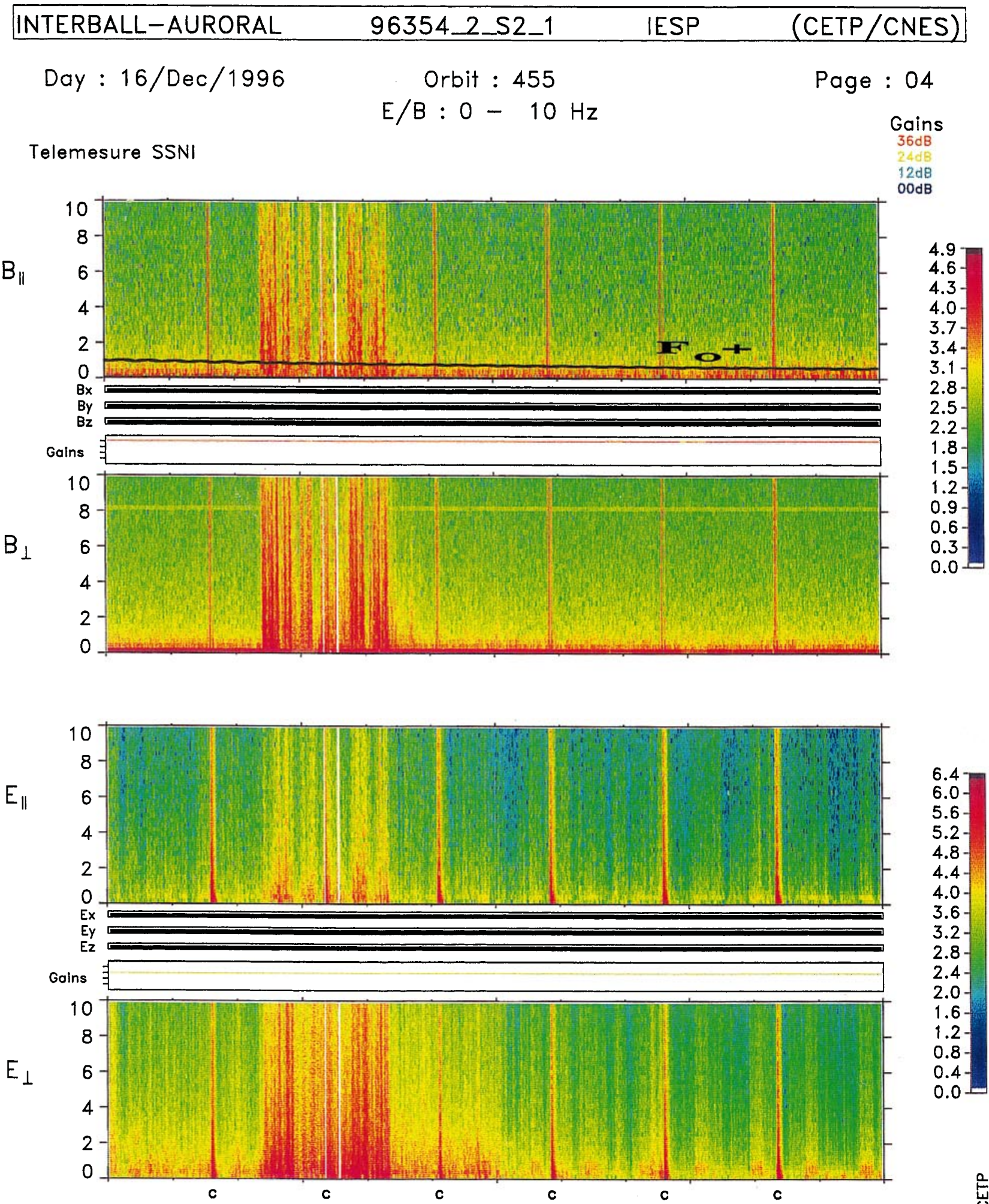

6.4
$6.0-$
5.6
$5.2-$
$4.8-$
$4.4-$
$4.0-$
$3.6-$
$3.2-$
$2.8-$
$2.4-$
$2.0-$
$1.6-$
$1.2-$
$0.8-$
$0.4-$
$0.0-$

UT $\quad 15: 00 \quad 15: 05 \quad 15: 10 \quad 15: 15 \quad 15: 20 \quad 15: 25 \quad 15: 30 \quad 15: 35 \quad 15: 40 \quad 15: 45 \quad 15: 50 \quad 15: 55 \quad 15: 59$

MLT $\quad 21: 37 \quad 21: 52 \quad 22: 07 \quad 22: 22 \quad 22: 36 \quad 22: 50 \quad 23: 03 \quad 23: 16 \quad 23: 29 \quad 23: 41 \quad 23: 52 \quad 00: 03 \quad 00: 14$

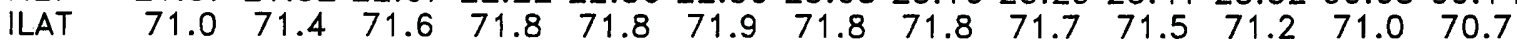

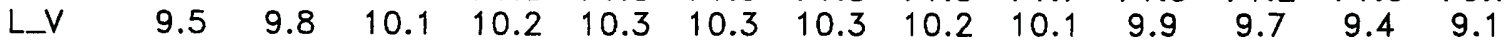

$\begin{array}{llllllll}\text { ALT } & 16002.7 & 16854.8 & 17568.9 & 18149.9 & 18600.7 & 18924.4 & 19123.0\end{array}$

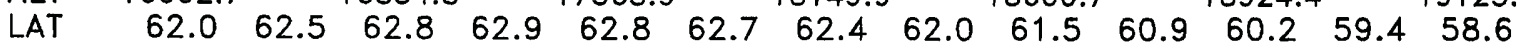

LONG $\quad 92.9 \quad 96.4 \quad 99.8 \quad 103.1 \quad 106.3 \quad 109.4112 .3115 .0117 .5119 .8122 .0124 .0125 .8$ 


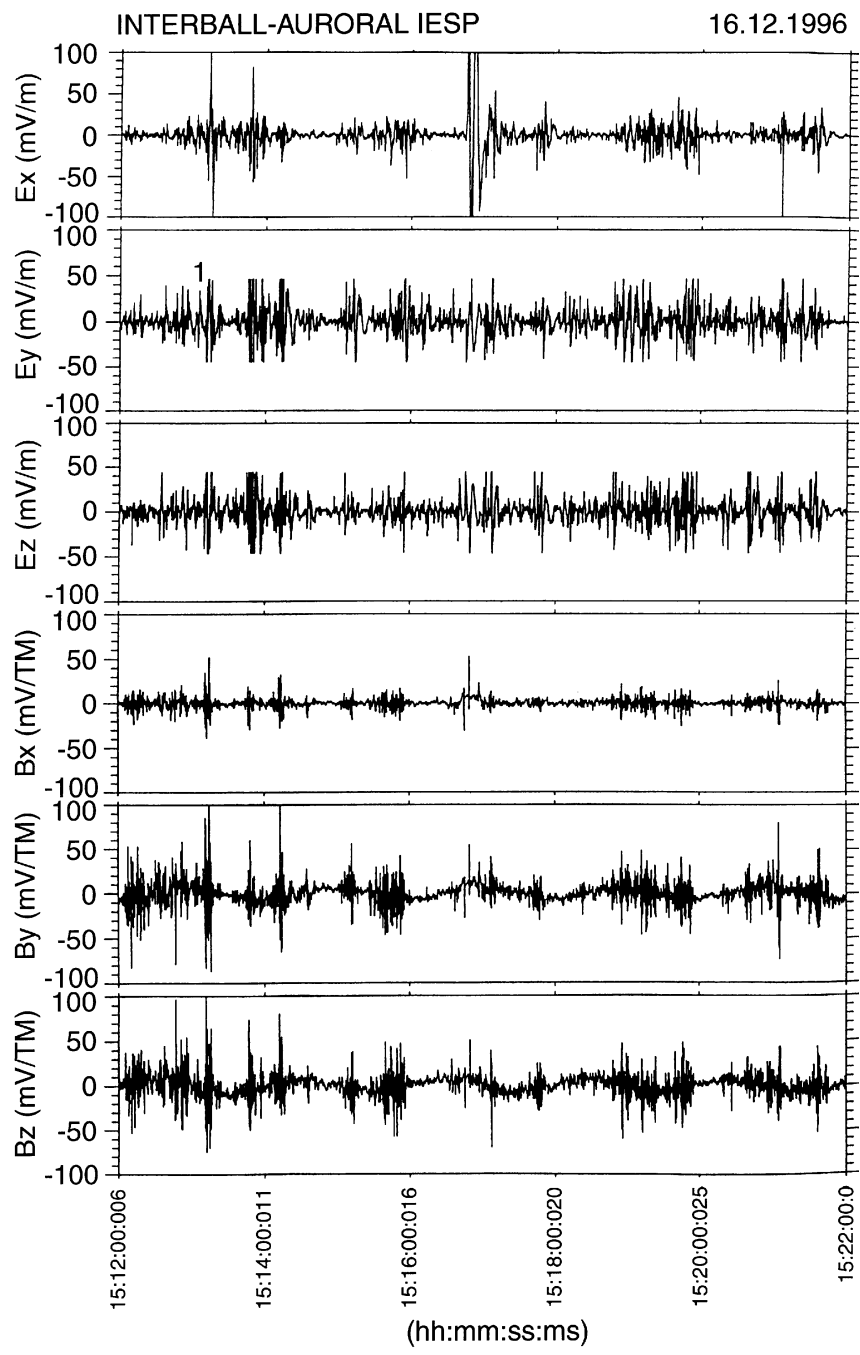

Fig. 4. Same as Fig. 1 for the event displayed in Plate 2. The intensification of the waves occurs simultaneously on the six components. The emission consists of successive wave packets more or less regularly spaced. The strong signal at $\sim 15: 16: 30$ UT is due the calibration of $\mathrm{NVKONCH}$

between 21:33:00 and 21:36:30 exhibits electrostatic emissions. This example shows clearly that the wideband fluctuations can be either electromagnetic or electrostatic and that the change from one regime to another is continuous. The kind of waves depends on the interacting plasma. The assessment of whether the observed waves are either electromagnetic or electrostatic needs a more systematic analysis based on the comparison with the distribution functions of particles. Nevertheless, at this stage of the study the role played by the heavy ions will be examined in the next section.

Figure 7 displays $1 \mathrm{~min}$ of signal. It is a good illustration of the difference between the behavior of the electric and of the magnetic components when one studies in great detail the waveforms, in particular the electric components are richer in higher frequencies around 21:40:30 UT. Moreover it shows a clear signature of a thin current structure at 21:40:35; the size of this filamentary current is estimated to $1-10 \mathrm{~km}$. At this stage of the study we cannot give its direction but

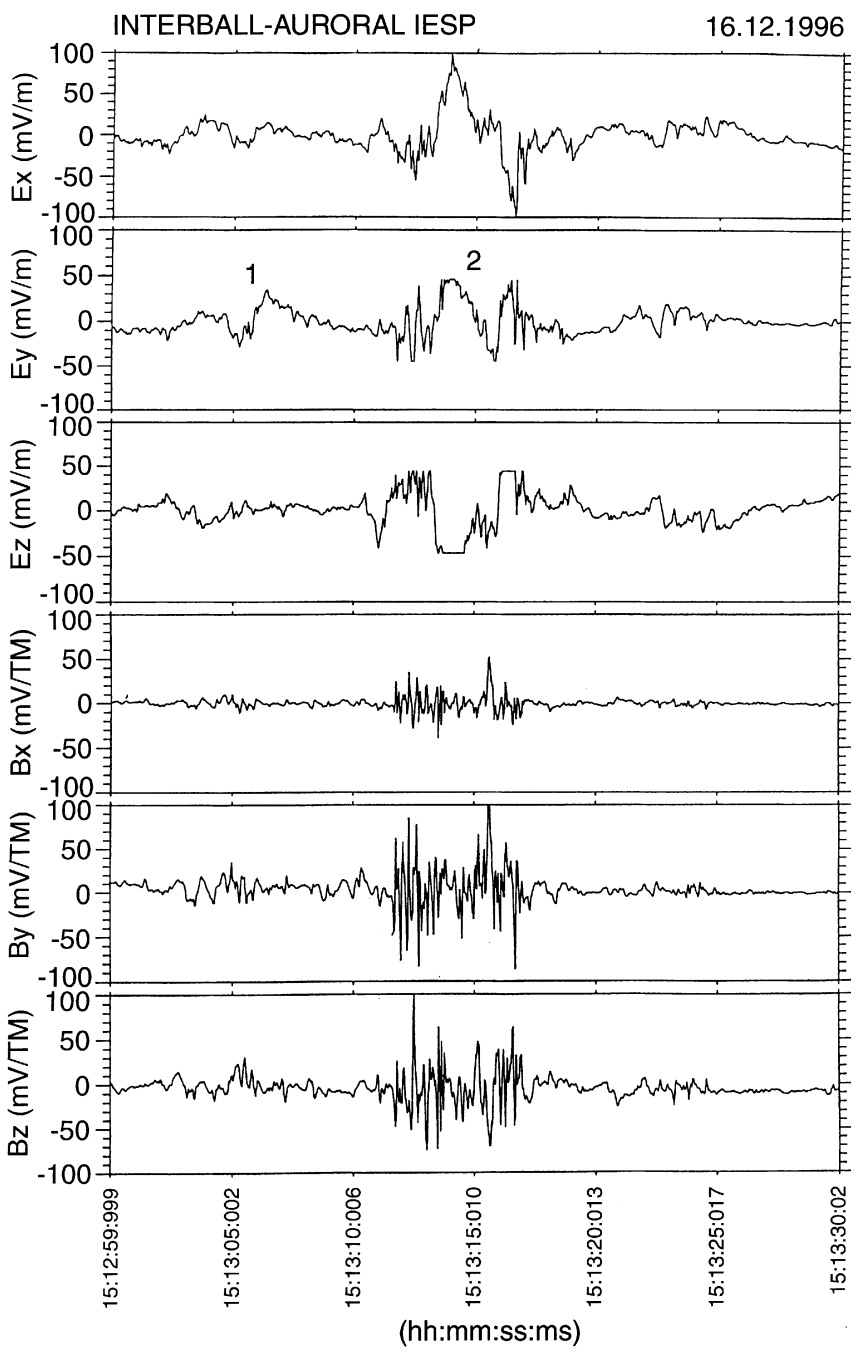

Fig. 5. Same as Fig. 4 with an extended scale. The emission is composed of fluctuations at different frequencies in agreement with the Plate 2 spectrograms

it will be done as the data will be transformed in a geophysical frame of reference.

Even if the data cannot yet be processed in the best frame of reference, the preliminary observations described enable us to propose interpretations of the mechanisms developing in the high-altitude auroral region.

\section{Interpretation}

Emissions observed in the auroral region at $\sim 1-2 R_{E}$ in the ULF frequency range have been already studied with the S3-3 satellite by Kintner et al. (1978), and Temerin and Lysak (1984) and more recently with Viking by Gustafsson et al. (1990). They have shown that these waves are generated by auroral electron beams near or below the altitude at which they are accelerated. The mechanisms described by these authors require the presence of heavy ions such as Helium. As the waves observed on board Interball have a part of their energy at a frequency well below the Helium 


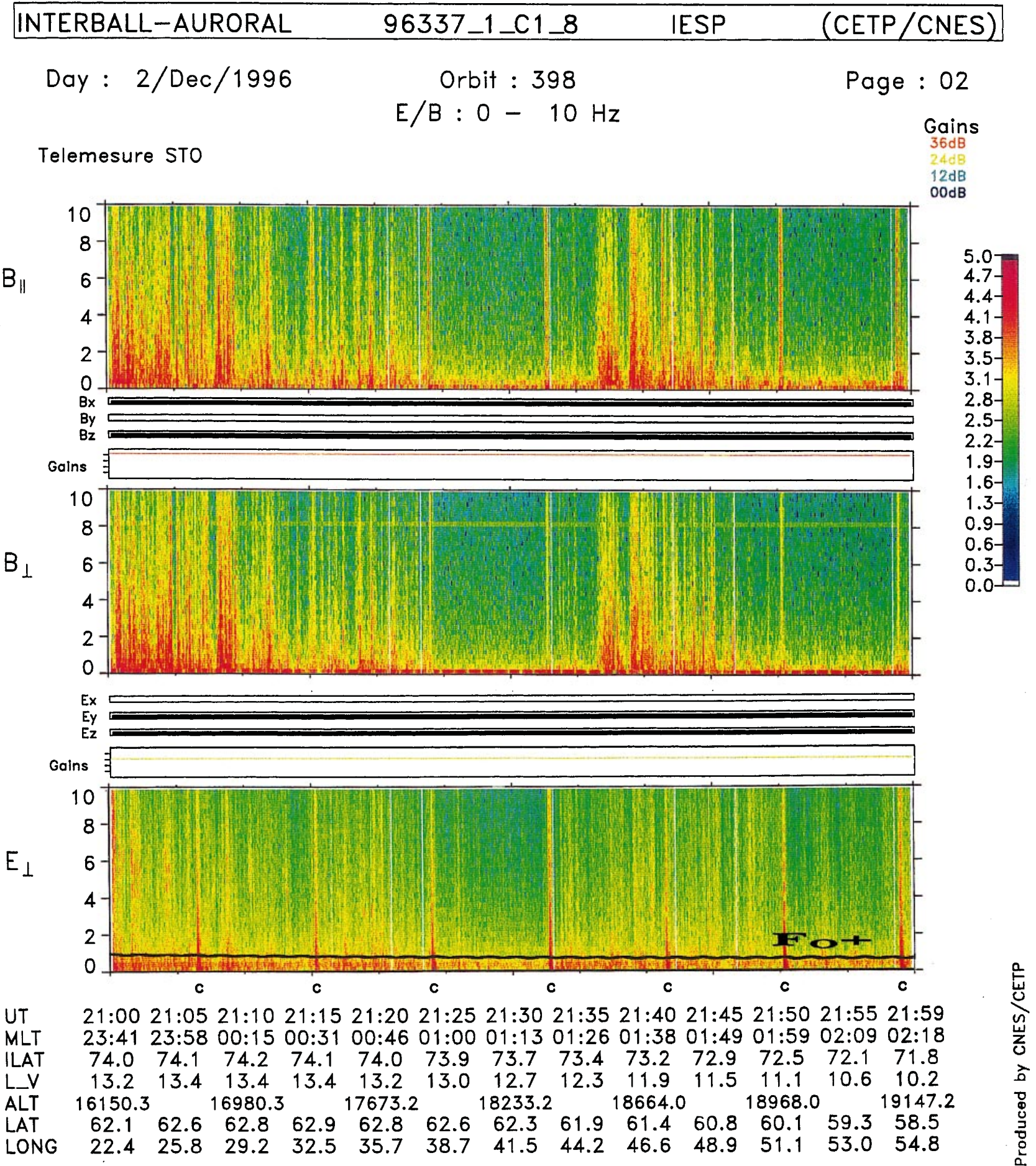

Processing Data Version : v1 - Plotting Dato Version : v1 - Date : 16/July/1997

Plate 3. Same as Plates 1 and 2. During this event the magnetic signal vanishes between 21:33 and 21:37 UT as it is also seen in Fig. 6

gyrofrequency we have extended the study of electron current-driven instabilities developed by Kindel and Kennel (1971) and Forslund et al. (1979) by considering the possible role of oxygen ions $\mathrm{O}^{+}$in this mechanism. The ion acoustic waves have been disregarded since they are strongly damped as soon as $T_{e}<T_{i}$, respectively the electron and ion thermal temperatures. We have revisited the work of Kindel and Kennel (1971) who have studied the electrostatic instability in single and multi-ion plasmas. They have found that (1) the electrostatic ion cyclotron waves are easily destabilized for a wide range of $T_{e} / T_{i}$, and (2) the heavy ion cyclotron wave is ordinarily the most unstable. Then Forslund et al. (1979) considered the electromagnetic regime of this instability and demonstrated that for $T_{e} \sim T_{i}$ the electromagnetic ion cyclotron instability 


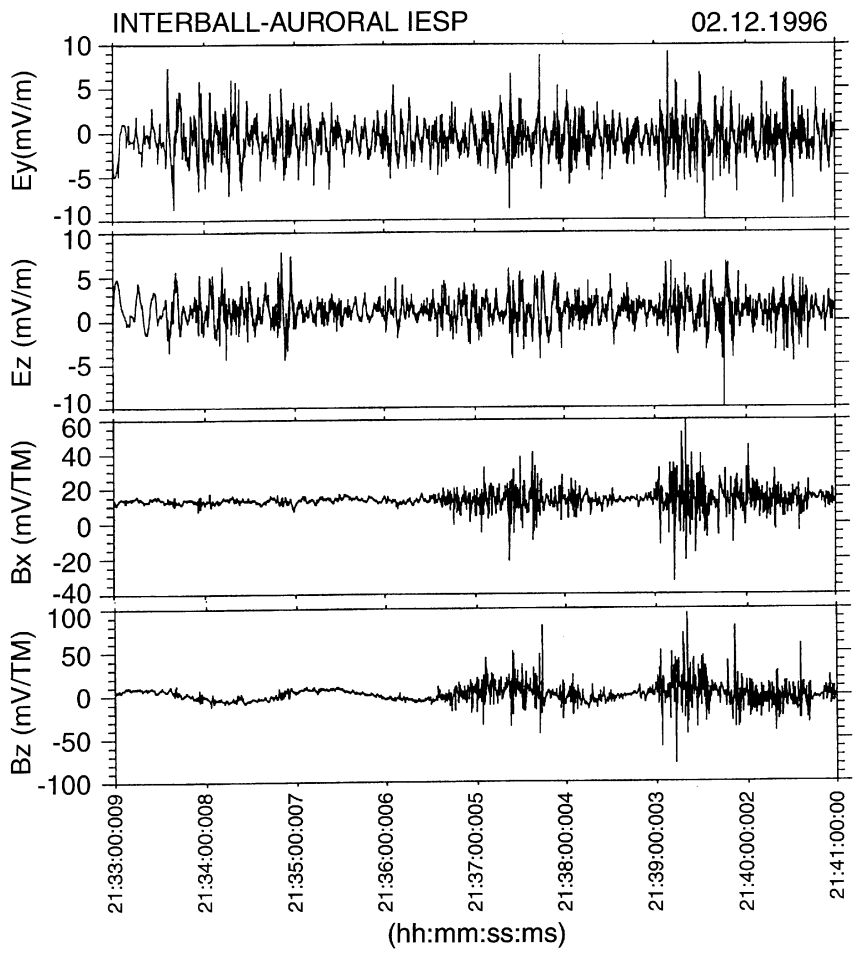

Fig. 6. Same as Fig. 1 for the event displayed in Plate 3. Notice that during this time-interval the magnetic components vary in intensity whereas the electric ones do not

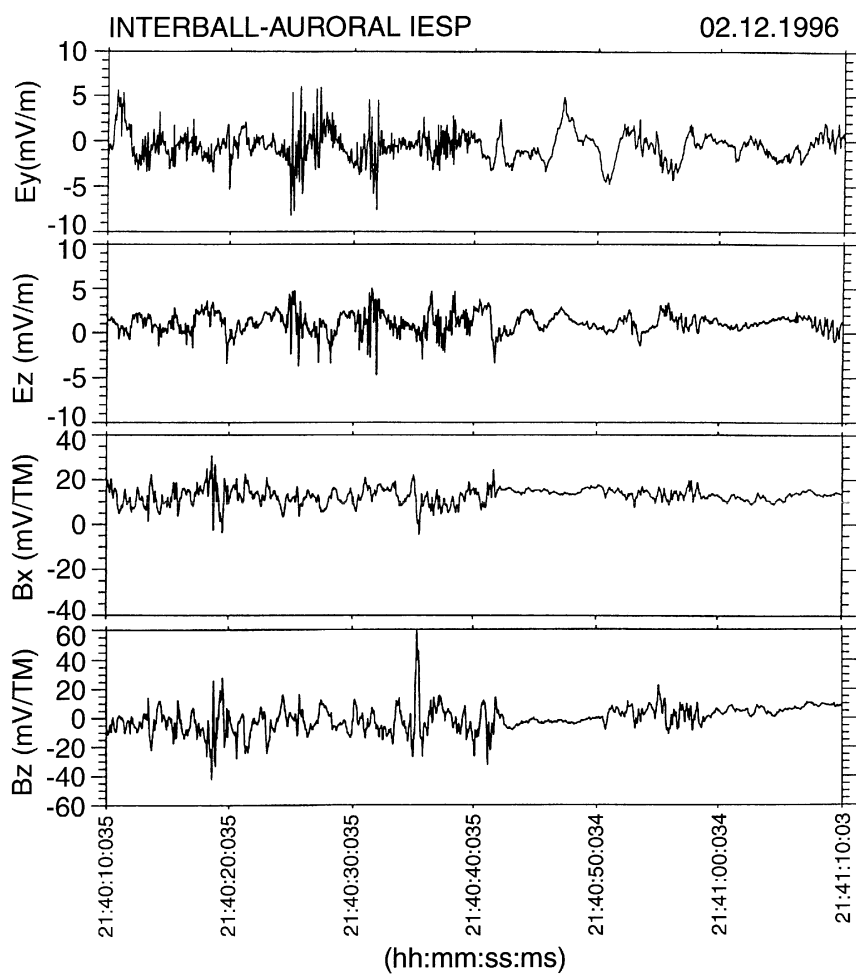

Fig. 7. Same as Fig. 6 with an extended time-scale. The electric components exhibit higher frequencies than the magnetic ones. A strong peak of short duration, at 21:40:35 UT, stands out from the other fluctuations on the magnetic component begins to have a lower threshold in terms of critical drift velocity $V_{d}$ than the corresponding electrostatic instability, as soon as $\beta_{i} \geq m_{e} / M_{i}$. Thus in a moderately high $\beta_{i}$, homogeneous collisionless plasma, the electromagnetic ion cyclotron instability is likely to have the lowest threshold of any electrostatic current-driven instability. Similar results can be found easily now by solving numerically the wave dispersion relation: we have used the computer code WHAMP (Rönnmark, 1983), which solves the dispersion relation of linear waves in a uniform magnetic field. To distinguish electrostatic (ES) from electromagnetic (EM) waves we have compared the ratio $\delta E / \delta B$ to $V_{A}$; for the sake of simplicity we define ES waves by $\delta E / \delta B>\mathrm{V}_{A}$ and EM waves by $\delta E / \delta B \leq \mathrm{V}_{A}$. As expected ES waves have much larger $k_{\perp}$.
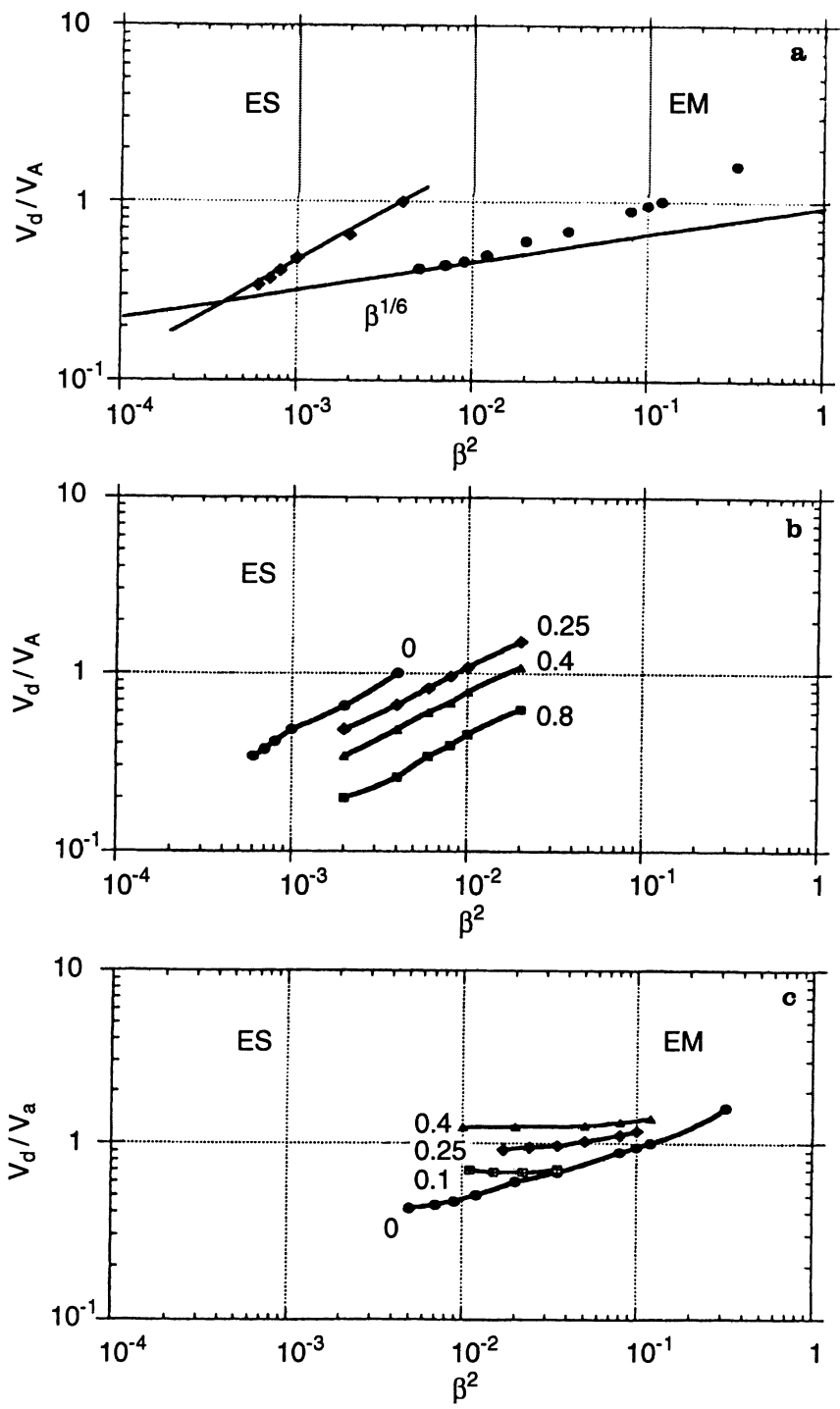

Fig. 8a-c. Marginal stability contours for the current-driven electrostatic and electromagnetic ion cyclotron instabilities. In abscissa, $\beta_{i}$, in ordinate the relative drift velocity between ions and electrons normalized to the Alfvén velocity. a ES and EM modes without addition of heavy ions; thin lines are the extrapolation of the corresponding curves; $\mathbf{b}$ and $\mathbf{c}$ for different $\mathrm{O}^{+}$concentrations: 0.1 , $0.25,0.4,0.8$ 
The results obtained by using WHAMP are similar to those found by Forslund et al. (1979) for a single ion $\left(\mathrm{H}^{+}\right)$plasma; they are displayed in Fig. 8a. For $M_{i} /$ $m_{e}=1836$ and $T_{e} / T_{i}=1$, the marginal stability contours $(\gamma \sim 0)$ have been plotted in $\left(\beta_{i}, V_{d} / V_{A}\right)$ parameter space. The contours $(\gamma \sim 0)$ have been drawn respectively for the electromagnetic EM and electrostatic ES ion cyclotron waves. The marginal stability curves have been extrapolated for low $\beta_{i}$. The marginal stability contour of the EM mode varies as $\beta^{1 / 6}$ for low $\beta_{i}$. When $\beta_{i}<m_{e} / M_{i}$ the critical drift velocity is lower for the ES mode than for EM mode and the frequency of the amplified wave is $\sim 1.2 F_{\mathrm{H}}+$. For $\beta_{i}>2 \cdot 10^{-2}$ the critical drift velocity of the EM mode varies linearly with the ion thermal velocity and the frequency of the amplified wave is $\sim 0.4 F_{H}+$.

Then the role of heavy ions on these instabilities was studied. The same code was applied to an $\mathrm{O}^{+}-\mathrm{H}^{+}$ mixture to calculate the critical drift velocity as a function of fractional $\mathrm{O}^{+}$density. We still assumed that $T_{e} / T_{i} \sim 1$ for the electron and $\mathrm{H}^{+}$populations, but since the $\mathrm{O}^{+}$population comes from the ionosphere, we took a low temperature $(1-10 \mathrm{eV})$ for that species. Figure $8 \mathrm{~b}$ and $\mathrm{c}$ display the marginal stability curves for ES and EM waves respectively for different oxygen concentrations. The two kinds of instability behave in opposite ways: the threshold of the drift velocity of the ES mode decreases when the concentration of $\mathrm{O}^{+}$increases, whereas the drift velocity of the EM mode increases. It is interesting to notice that the group velocity parallel to the field line for the EM waves is very large with a single ion $\left(\mathrm{H}^{+}\right)$and decreases sharply with the addition of $\mathrm{O}^{+}$.

Therefore the ES mode develops more easily when $\mathrm{O}^{+}$is present in the medium. In such conditions the frequency which is excited is close to the bi-ion hybrid frequency. Thus the electrostatic quasi-monochromatic waves described in Sect 3.1 could be the result of such an interaction; accordingly the measurement of its frequency would be an indirect measurement of the composition of the medium. This condition is reinforced by the fact that these waves are locally generated. The interpretation of the wide-band emissions is not so straightforward and requires discussion.

\section{Discussion}

The current-driven instability studied in the previous section is a good candidate for interpreting most of the phenomena described in Sect. 3. The quasi-monochromatic emissions above the $\mathrm{O}^{+}$gyrofrequency are electrostatic and can easily be amplified, especially when $\mathrm{O}^{+}$is present. As a matter of fact, most of the time the particle experiments detect during these events an increase in the flux of oxygen, which favors this interpretation.

The electromagnetic emissions with a wide band spectrum are clearly associated with electron precipitation; thus they could also be due to a current-driven instability: as discussed in the previous section electromagnetic current-driven instability is likely to develop in a regime where the protons are the dominant species and $\beta$ is large enough. Although the emission amplified at a given point along the field line will have a characteristic frequency which depends on the plasma condition at this altitude, the waves will propagate more or less guided along $B_{0}$, thus Interball will detect waves which are destabilized at different altitudes and the spectrum will correspond to the summation of different frequencies coming from different altitudes. A similar interpretation has been invoked by several studies for explaining electromagnetic waves observed at low altitude with satellites or rocket experiments. For example Lund and LaBelle (1997) have shown that the source of the waves detected in the auroral ionosphere $(\sim 400 \mathrm{~km})$ are electromagnetic ion cyclotron waves generated at much higher altitudes $(\sim 5000 \mathrm{~km})$.

It is more difficult to invoke the same argument for the electrostatic wide-band waves since their group velocity is much smaller than for the electromagnetic ones; Plate 1 shows electrostatic wide-band emissions superimposed on quasi-monochromatic structures; we suggested previously that the broadening of the spectrum could be a Doppler broadening. The results of a detailed analysis of this event are shown in Figs. 9 and 10. The signal of $E_{X}$ component has been filtered respectively in the range corresponding to the occurrence of the quasi-monochromatic structures (between 1 and $3.3 \mathrm{~Hz}$ ) and in the range above $6 \mathrm{~Hz}$. They are plotted in Fig. 9, at the same scale as the initial waveform. The amplitude of the signal in the range above $6 \mathrm{~Hz}$ is well below the amplitude of the structured emissions; but the intensification of the waves above $6 \mathrm{~Hz}$ is proportional to the signal in the intermediate range; then the link between the signals at different frequencies suggests that we are detecting the same fluctuations which are broadened by a strong Doppler effect. In Fig. 10 the spectra of the waves at two different time-intervals are plotted. In Fig. 10a, a clear peak superimposed on a broad spectrum is identified. In Fig. 10b the monochromatic emission disappears but the slope of the broad-band emission remains the same. The similarity between the two slopes of the spectra is another indication for the Doppler shift. Therefore the electrostatic wide-band emissions could be simply due to the Doppler broadening of monochromatic emissions which are not systematically observed; the monochromatic emissions can be either swamped in the Doppler broadening or have a very low frequency $(\ll 1 \mathrm{~Hz})$. For example one observes on the $E_{\perp}$ component a signal below $\mathrm{F}_{\mathrm{O}}+$ still presents between 21:33:00 and 21:36:30 when the emission is electrostatic. A rough estimate of the Doppler broadening can be obtained by setting:

$$
\frac{k V}{\omega_{0}}=k_{\perp} \rho_{i} \frac{V}{V_{\mathrm{thi}}} \frac{\Omega_{H+}}{\omega_{0}}
$$

$V$ is the relative spacecraft plasma velocity of $15 \mathrm{~km} / \mathrm{s}$ taken from the HYPERBOLOÏD experiment (N. Dubouloz, private communication); $\rho_{i}$ is the ion Larmor radius, and from the resolution of the dispersion relation, one gets an estimate of $k_{\perp} \rho_{i} \sim 2$; assuming that the ion thermal velocity is $\sim 100 \mathrm{eV}$, the Doppler shift is 

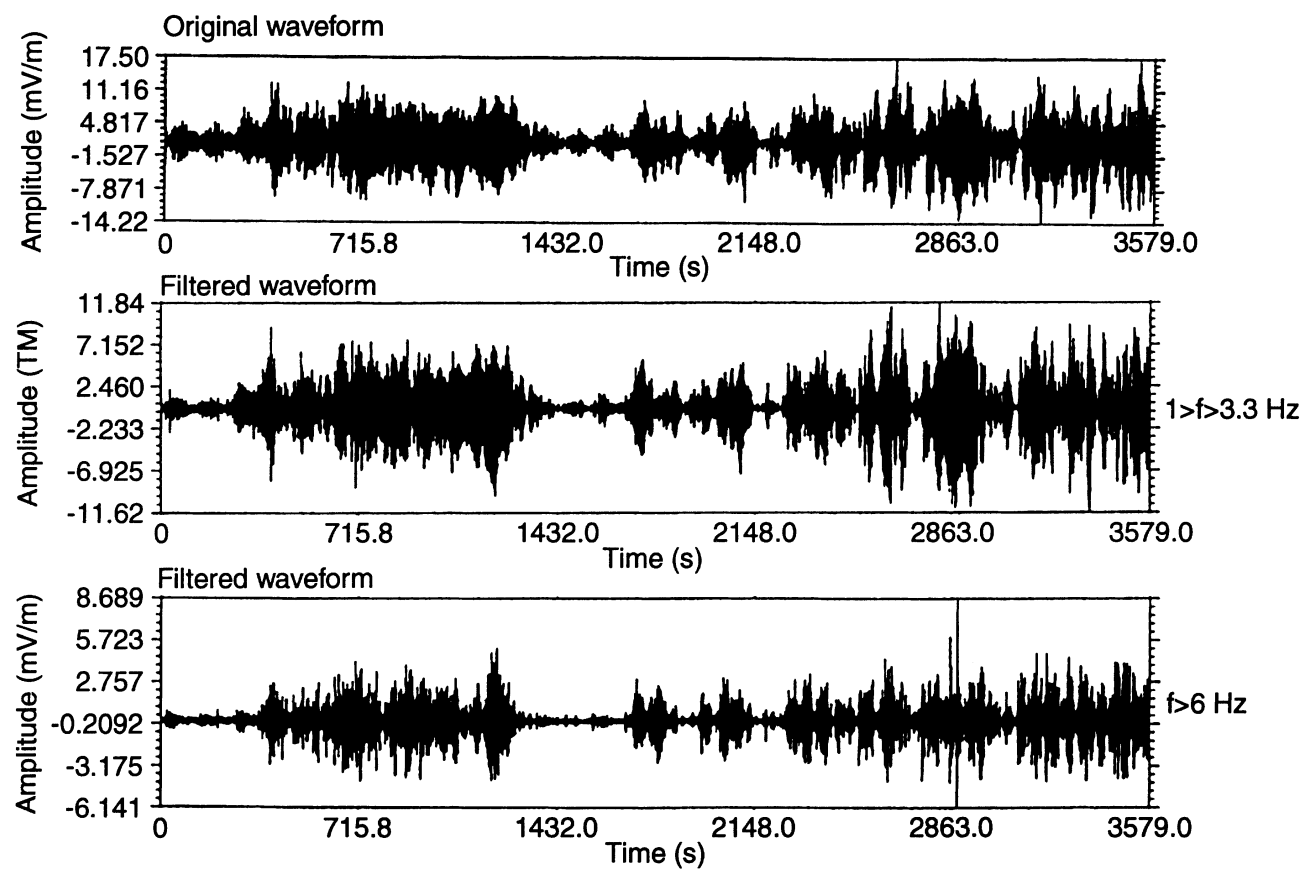

Fig. 9. Waveforms of the $E_{X}$ component for the event displayed in Plate 1. On the top initial signal, in the middle the signal is filtered between 1 and $3.3 \mathrm{~Hz}$, in the lower part the signal is filtered above
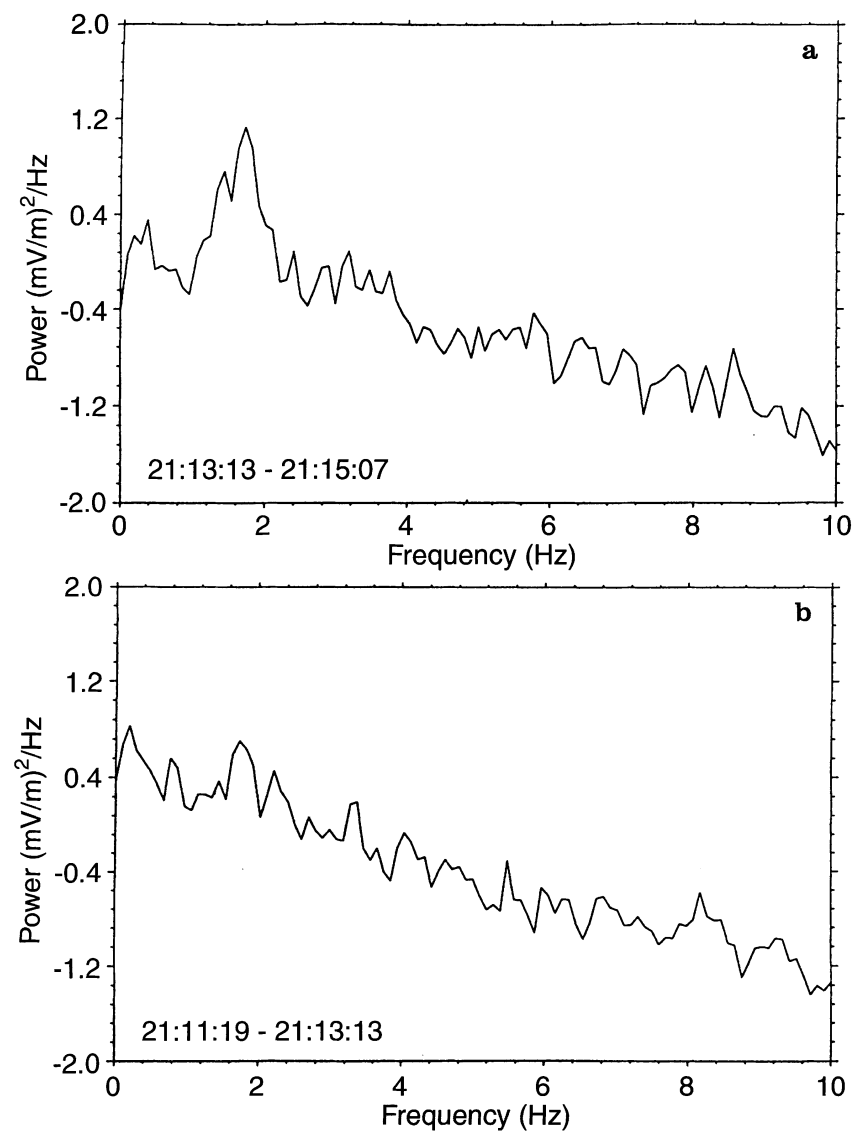

Fig. 10. Same as Fig. 3 for other time-intervals. The waves are very intense and the slopes of the spectra are very similar. At 21:11:19 UT the quasi-monochromatic wave is not clearly seen since the broadening of the emission spreads out below and above the amplified signal
$6 \mathrm{~Hz}$. The signal in the lowest panel occurs only when the signal in the middle panel intensifies

of the order of $200 \%$. In fact $k_{\perp} \rho_{i}$ has been underestimated since it is derived from the marginal instability condition. Then the Doppler broadening is probably much larger and could explain the width of the spectra.

Since the value of $k_{\perp}$ for the EM instability is at least ten times less than for the ES mode, we do not expect a large Doppler shift to occur for the EM waves. The two kinds of emission are guided along the field lines but the group velocity of the EM waves is much faster. Then the interpretation based on propagation effect already mentioned is more plausible.

\section{Conclusion}

Thanks to the magnetic and electric sensor implemented onboard the Interball Auroral probe, both electrostatic or electromagnetic waves have been detected in the auroral region above $2 \mathrm{R}_{\mathrm{E}}$. From an observational point of view one distinguishes two kinds of fluctuations: (1) electrostatic emissions which consist of quasi-monochromatic structures above the oxygen gyrofrequency, superimposed on a wide band signal interpreted as a Doppler broadening, (2) electromagnetic fluctuations with a wide band spectrum and a very bursty character.

The observations are interpreted as electromagnetic EM and electrostatic ES ion cyclotron waves which can be unstable to field-aligned currents. These instabilities have been studied in detail; Forslund et al. (1979) have demonstrated that for $T_{e} \sim T i$, the EM instability begins to have a lower threshold of the critical drift velocity than the corresponding ES instability for $\beta_{I} \geq$ $m_{e} / M_{i}$. At lower $\beta_{i}$ the critical drift velocity of the ES waves is lower and the ES waves are preferentially 
amplified. Moreover the thresholds for ES and EM behave in opposite way in an $\mathrm{O}^{+}$-rich plasma: the threshold for the critical drift velocity between ions and electrons for the ES waves is lowered by increasing the fractional density $\mathrm{O}^{+}$whereas it increases for the EM waves. Then from this study it is obvious that the $\beta_{i}$ parameter and the density of the heavy ions play a crucial role in controlling this kind of instability. In summary, one expects a currentdriven ES emissions for low $\beta_{i}$ plasma $\left(\beta_{i}<m_{\mathrm{e}} / M_{i}\right)$ and/or in presence of $\mathrm{O}^{+}$, and EM waves when $\beta_{i}$ is larger and with few $\mathrm{O}^{+}$ions. A more detailed study of these two kinds of EM or ES fluctuations needs a more extensive analysis, especially a detailed comparison with particle distributions measured simultaneously.

Acknowledgements. The authors thank the Magnetometer team for providing us their data. The authors are also grateful to the large number of people who have contributed to the success of the Interball project. In particular, we want to acknowledge the operational teams, especially at the Space Research Institute in Moscow for managing the launch and the telemetry system in a difficult situations, and the eminent work in instrument performed by LBElectronics in Hungary. This work has been supported by CNES which has provided funds and support for the data processing.

Topical Editor K.-H. Glassmeir thanks A. Coates for his help in evaluating this paper.

\section{References}

Boström, R., G. Gustafsson, B. Holback, G. Holmgren, H. Koskinen, and P. Kintner, Characteristics of solitary waves and weak double layers in the magnetospheric plasma, Phys. Rev. Lett., 61, 82, 1988.

Chmyrev, V. M., S. V. Bilichenko, O. Pokhotelov, V. A. Marchenko, V. I. Lazarev, A. V. Streltsov, and L. Stenflo, Alfvén vortices and related phenomena in the ionosphere and the magnetosphere, Phys. Scr., 38, 841, 1988.

Forslund, D. W., J. M. Kindel, and M. A. Stroscio, Current driven electromagnetic ion cyclotron instability, J. Plasma Phys., 21, $127,1979$.

Goertz, C. K., Electron acceleration via kinetic Alfvén waves, in Comparative study of magnetospheric systems, CepaduesEditions, Toulouse, France, p. 357, 1986.
Gustafsson, G., M. André, L. Matson, and H. Koskinen, On waves below the local proton gyrofrequency in auroral acceleration regions, J. Geophys. Res., 95(A5), 5889, 1990.

Hasegawa, A., and C. Uberoi, The Alfvén wave, DOE Critical Review Series, DOE/TIC-1197, 1982.

Kindel, J. M., and C. F. Kennel, Topside current instabilities, $J$. Geophys. Res., 76, 3055, 1971.

Kintner, P. M., M. C. Kelley, and F. S. Mozer, Electrostatic hydrogen cyclotron waves near one earth radius altitude in the polar magnetosphere, Geophys. Res. Lett., 5, 139, 1978.

Lefeuvre, F., M. Parrot, M. Mogilevsky, J. L. Rauch, B. Poirier, J. P. Dume, P. Fergeau, M. Leveque, P. Martin, J. M. Moreau, and P. Zamora, Multicomponent wave measurements on-board the auroral satellite of the Interball project: the MEMO experiment, in Interball mission and payload, Eds. RK, IKI, CNES, Toulouse, p. 359, 1995.

Lindqvist, P. A., and G. T. Marklund, A statistical study of highaltitude electric fields measured on the Viking satellite, $J$. Geophys. Res., 95, 5867, 1990

Louarn P., A. Roux, H. de Féraudy, D. Le Quéau, M. André, and L. Matson, Trapped electrons as a free energy source for the auroral kilometric radiation, J. Geophys. Res., 95(A5), 5983, 1990

Louarn, P., J. E. Wahlund, T., Chust, H. de Féraudy, A. Roux, B. Holback, P. O. Dovner, A. I. Eriksson, and G. Holmgren, Observation of kinetic Alfvén waves by the FREJA spacecraft, Geophys. Res. Lett., 21, 1847, 1994.

Lund, E. J., and J. LaBelle, On the generation and propagation of auroral electromagnetic ion cyclotron waves, J. Geophys. Res., 102 (A8), 17241, 1997.

Mozer, F. S., and M. Temerin, Solitary waves and double layers as the source of parallel electric fields in the auroral acceleration region in High-latitude space plasma physics, Eds. B. Hultqvist and T. Hagfors, Plenum, New York, p. 453, 1983.

Riedler, W., K. M. Torkar, A. Pedersen, R. Schmidt, K. G. Rudenauer, M. Fehringer, and Yu. I. Galperin, Spacecraft potential control experiment RON, in Interball mission and payload, Eds. RK, IKI, CNES, Toulouse, p. 373, 1995.

Rönnmark, K., Computations of the dielectric tensor of a Maxwellian plasma, Plasma Phys., 25, 699, 1983.

Temerin M., and R. Lysak, Electromagnetic ion cyclotron mode (ELF) waves generated by auroral electron precipitation, $J$. Geophys. Res., 89(A5), 2489, 1984.

Volokitin, A. S., and E. M. Dubinin, The turbulence of Alfvén waves in the polar magnetosphere of the Earth, Planet. Space Sci., 37, 761, 1989. 